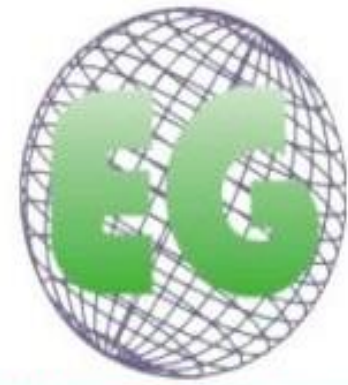

ISSN 1695-6141

$N^{\circ} 46$

\title{
Perfil socio-demográfico de los cuidadores de los pacientes geriátricos hospitalizados mayores de 75 años y su relación con la satisfacción
}

Socio-demographic profile of caregivers of geriatric hospital patients over 75 years and its relationship with satisfaction

\begin{abstract}
*Hanzeliková, Alika **López-Muñoz, Francisco ***Fusté Moreno, Rafael
*Servicio de Salud de Castilla La Mancha (SESCAM), Complejo Hospitalario de Toledo. **Cátedra de Medicina Genómica. Facultad de Ciencias de la Salud, Universidad Camilo José Cela. Instituto de Investigación Hospital 12 de Octubre (i+12). Madrid. E-mail: flopez@ucjc.edu ***servicio de Urgencias.
\end{abstract} Unidad de Enfermedades Respiratorias. Hospital Lleuger de Cambrils Antoni de Gimbernat. Tarragona. España.

http://dx.doi.org/10.6018/eglobal.16.2.249861

\section{RESUMEN}

Objetivo: Determinar/establecer la relación entre los factores socio-demográficos y las dimensiones de calidad de atención del cuidado y satisfacción en los cuidadores de los pacientes geriátricos agudos ingresados en el Hospital Virgen del Valle (Toledo, España).

Metodología: Diseño: Estudio descriptivo transversal. Ámbito de estudio: Hospital geriátrico de Toledo (España).Sujetos de estudio: Cuidadores principales de los pacientes geriátricos ingresados en el Hospital Virgen del Valle de Toledo, en las Unidades de Agudos. Tamaño muestral: 267 cuidadores ( $\mathrm{p}=$ 0.5 , intervalo de confianza del $95 \%$, e= 0.06). Instrumento de medida; Cuestionario validado, basado en el modelo SERVQUAL, para medir la satisfacción con la atención recibida. Determinaciones: Para determinar el efecto de las variables predictoras en el nivel de satisfacción, se realizaron análisis de regresión lineal simple y multivariante.

Resultados: La valoración global sobre la satisfacción general con la atención y con los cuidados es "mediatamente buena", siempre obteniendo puntuación más alta en la valoración de los cuidados que en la atención en general. En relación con los ítems estudiados sobre la satisfacción y sobre las dimensiones de calidad y perfil del cuidador, se han encontrado diferencias estadísticamente significativas en: género, edad, nivel de estudios del cuidador, tiempo de hospitalización, nivel de dependencia del enfermo, comparten domicilio o no, e ingresos anteriores.

Conclusión-Esencia: Existe una necesidad urgente de aumentar el nivel de conocimiento sobre los cuidadores y conocer sus expectativas sobre el cuidado, para elaborar estrategias de cuidado acordes 
con sus preferencias. El conocimiento de las deficiencias detectadas sobre el cuidado proporciona a los profesionales datos sobre donde se debe intervenir para conseguir cambios favorables para aumentar la satisfacción.

Palabras clave: satisfacción; cuidador principal; calidad de cuidados; geriatría; pacientes hospitalizados.

\section{ABSTRACT}

Purpose: To determine / establish the relationship between socio-demographic factors and dimensions of care quality of care and satisfaction in caregivers of elderly patients admitted to acute Hospital Virgen del Valle (Toledo, Spain).

Methodology: Design: Cross-sectional study. Field of study: Geriatric Hospital of Toledo (Spain). Study subjects: Main caregivers of elderly patients admitted to the Hospital Virgen del Valle de Toledo, in the acute care setting. Sample size: 267 caregivers $(p=0.5$, confidence interval $95 \%$, e $=0.06$ ). Measuring instrument; Validated questionnaire, based on the SERVQUAL model to measure the satisfaction of the care provided. Determinations: To determine the effect of the predictor variables on the level of satisfaction, simple and multivariate analyzes were performed linear regression.

Results: The overall assessment of satisfaction with the attention of care is "immediately good", always getting higher scores on the assessment of care in care in general. In relation to the items surveyed on satisfaction and quality dimensions and caregiver profile, found statistically significant differences in gender, age, education level of the caregiver, hospitalization time, level of dependence of the patient, share home or not, and previous earnings.

Essence /Conclusion: There is an urgent need to increase the level of awareness of carers and meeting their expectations for care, care to develop strategies according to their preferences. Knowledge of deficiencies on caring professionals provides data on where to intervene to achieve favorable changes to increase satisfaction.

Keywords: satisfaction; primary caregiver; quality of care; geriatric patients.

\section{INTRODUCCIÓN}

El envejecimiento humano es un fenómeno universal e inevitable. La población mayor de 65 años (inclusive) alcanza ya los 380 millones de personas en el mundo, y para el año 2020, se prevé que aumente hasta los 690 millones ${ }^{1-2}$. España es uno de los países más envejecidos en la Unión Europea ${ }^{3}$, con más de 7,5 millones de personas de más de 65 años. Las personas de edad avanzada ya representan el $17 \%$ de toda la población, y ese porcentaje no va a dejar de crecer en las próximas décadas, y según las últimas estimaciones puede llegar hasta el $37,85 \%$ en el año $2051^{4}$. Este aumento de la población mayor produce un incremento de la demanda de atención sanitaria, dado que en esta etapa se es más vulnerable que en otras edades a la enfermedad ${ }^{5}$.

Aproximadamente el $80 \%$ de las personas mayores presentan al menos una enfermedad crónica, y el $50 \%$ dos. El promedio es de 2,8 problemas o enfermedades crónicas en personas entre 65 y 74 años, llegando a 3,23 en personas mayores de 75 años. Estas patologías crónicas son, en gran parte, las causantes de las limitaciones en la actividad y de la discapacidad ${ }^{5}$. En las personas mayores de 85 años, los ingresos hospitalarios son 10 veces mayores que en las edades comprendidas entre los 15 y 34. De la misma forma, la estancia media de cada ingreso aumenta en grupos de personas de edad avanzada ${ }^{6}$. Algunos estudios demuestran que en España, los pacientes mayores de 85 años tienen un índice de pluripatología del $95,1 \%{ }^{4}$. La Organización Mundial de la Salud ha estimado que las patologías 
crónicas (diabetes, enfermedades cardiovasculares, trastornos mentales, etc.) constituirán la principal fuente de discapacidad en $2020^{6}$.

Esta situación condiciona una mayor necesidad de cuidadores. Los servicios prestados por los cuidadores pueden garantizar una mejora de calidad de vida de estas personas. Los cuidadores pueden ser formales o informales, incluyendo entre estos últimos la familia, amigos, vecinos o sujetos de confianza que proporcionan cuidado no remunerado ${ }^{7}$. Los miembros de la familia entre los cuidadores informales son de suma importancia en la atención, sobre todo en la cultura hispana, en la que los lazos familiares y la unión es la norma. Los miembros de la familia ayudan en las actividades de la vida diaria y también proporcionan apoyo emocional a estas personas $^{8}$.

El valor económico de la prestación de cuidados informales es incalculable. A la hora de un ingreso hospitalario, el cuidador asume la responsabilidad de acompañamiento del enfermo geriátrico. En muchas ocasiones, estos enfermos pueden tener dificultades para expresar sus necesidades y percepciones acerca del cuidado. En estos casos, los familiares/cuidadores del paciente desempeñan un papel importante en los procesos del cuidado y de la atención en general. Frecuentemente la opinión de los familiares/cuidadores puede generar información útil sobre la calidad de la asistencia geriátrica. Los familiares/cuidadores sienten que es su responsabilidad supervisar y evaluar la calidad ${ }^{9}$.

El fenómeno de los cuidadores de ancianos ha sido estudiado en contextos diferentes, pero ha sido escasamente analizado como evaluador de la satisfacción y calidad de atención hospitalaria. En base a lo anterior, el presente estudio pretende conocer cuál es la calidad de la asistencia percibida por los cuidadores, y si tiene relación directa el perfil socio-demográfico de los cuidadores con el nivel de satisfacción en cuanto a la atención y cuidados recibidos. Este estudio pretende evaluar los problemas que perciben los cuidadores y las barreras que puede haber en el buen funcionamiento entre los cuidadores y la prestación de los cuidados. Una vez detectadas sus necesidades relacionadas con este perfil, se podrían implantar las medidas correctoras e innovadoras oportunas. Se considera que es una cuestión primordial la mejora de la integración de los cuidadores dentro de los espacios y estructuras del sistema hospitalario. Instaurar intervenciones basadas en el resultado de este estudio podrían concebirse para elevar la calidad de cuidados de las personas mayores y para reducir los resultados negativos.

\section{MATERIAL Y MÉTODOS}

Diseño: Estudio descriptivo transversal.

\section{Ámbito de estudio: Hospital Geriátrico de Toledo (España)}

Sujetos de estudio: Cuidadores principales de los pacientes geriátricos ingresados en el Hospital Virgen del Valle de Toledo en las Unidades de Agudos.

Tamaño muestral: Suponiendo que la estimación del porcentaje responda a una sola característica de respuesta dicotómica y el caso más desfavorable de $p=0,5$, para un intervalo de confianza de $95 \%$ y una precisión de $\pm 6 \%(e=0,06)$, se precisó una muestra aleatoria de 267 cuidadores. 
Criterios de inclusión:

- Persona que habitualmente permanece con el paciente.

- Haber permanecido en la unidad un mínimo de ocho días.

- Acceder voluntariamente a participar en el estudio.

- No presentar déficits cognitivos.

Criterios de exclusión:

- Negarse a participar en el estudio.

- Profesional sanitario o familiar de personal sanitario.

- Participación previa en el estudio por causa de reingreso.

Variables del estudio:

a) Variables independientes

a.1) Variables de control

a.1.1). Variables socio-demográficas

- Edad del cuidador y del paciente a quien cuida

- Género

- Comparten domicilio con el paciente

- Ingresos anteriores

- Nivel de estudios

- Situación laboral

- Tipo de relación con el enfermo

- Estancia en el hospital

a.1.2.) Variables clínicas del paciente

- Motivo de ingreso

- Nivel de conciencia

- Nivel de deterioro cognitivo

- Nivel de dependencia

- Dependencia de cuidados familiares previo al ingreso

b) Variables relacionadas con la calidad de los cuidados

- Dimensiones de calidad percibida

- Tangibilidad

- Fiabilidad

- Capacidad de respuesta

- Seguridad

- Empatía

c) Variable dependiente:

- Satisfacción global con la atención recibida en la Unidad y con los cuidados de enfermería. 
Instrumento de medida: Se utilizó un cuestionario validado, basado en el modelo SERVQUAL, adaptado a los cuidados. El cuestionario consta de 22 ítems agrupados en 5 dimensiones (tangibilidad, fiabilidad, capacidad de respuesta, seguridad y empatía), en dos bloques para las percepciones y expectativas. Los ítems se responden en una escala de 1-7. Se incluyó también una pregunta sobre el orden de importancia (de 1a 5) que tiene para los cuidadores las 5 dimensiones anteriormente mencionadas. Finalmente y siguiendo la pauta habitual en la mayoría de los cuestionarios de satisfacción, se incluyó una pregunta sobre la satisfacción general sobre los cuidados y una pregunta sobre la satisfacción global con la atención recibida, valorada con una escala de intervalo de 0 a 10.

Se realizó un análisis de resultados del pilotaje: comprensión de las preguntas, coherencia interna, adecuación y pertinencia de las variables y cálculo de indicadores de validez y fiabilidad (alpha de Cronbach $=0,918$ ).

Recogida de datos: Entrevista personal, anónima, confidencial.

Análisis estadístico: Las variables cuantitativas se describieron como media y desviación típica y las variables cualitativas mediante frecuencias absolutas y relativas. Para determinar el efecto de las variables predictoras en el nivel de satisfacción se realizaron análisis de regresión lineal simple y multivariante. El nivel de significación estadística se estableció en $p<0.05$.

Consideraciones éticas: El estudio fue aprobado por la Comisión de Investigación, por el Comité Ético de Investigación Clínica en el Complejo Hospitalario de Toledo y por la dirección del Hospital. Los participantes en el estudio fueron informados sobre el método y el fin de la investigación. Se respetó la libre participación en el estudio. Una vez obtenida la información se documentó de manera anónima, para ser empleada exclusivamente para los fines de la investigación.

Financiación externa: Fundación de Investigación Sanitaria de Castilla la Mancha.

\section{RESULTADOS}

La muestra de estudio está constituida por 267 cuidadores. El 22,8\% ( $n=60)$ fueron varones y el $77,7 \%(n=207)$ mujeres. La edad media fue de 59,39 años (DE = 13,46): 60,73 años (DE $=13,54$ ) en el caso de los varones y 59,02 años (DE $=12,92$ ) en las mujeres. Las edades de los encuestados oscilan entre 20 y 88 años.

Las Figuras 1 y 2 muestran la distribución de los cuidadores por edad y género. Se observa que en el rango de edad donde más cuidadores hay es entre 55 y 65 años en ambos sexos. Es en este mismo grupo donde hay más porcentaje de hombres. Donde menos hombres cuidadores se encuentran es en el rango de edad de 65 y 74 años. El resto de los cuidadores, según rango de edad y sexo es muy similar. En la Tabla 1 se pueden observar todas las variables socio-demográficas de los cuidadores. 
Figura 1: Distribución de los cuidadores por edad.

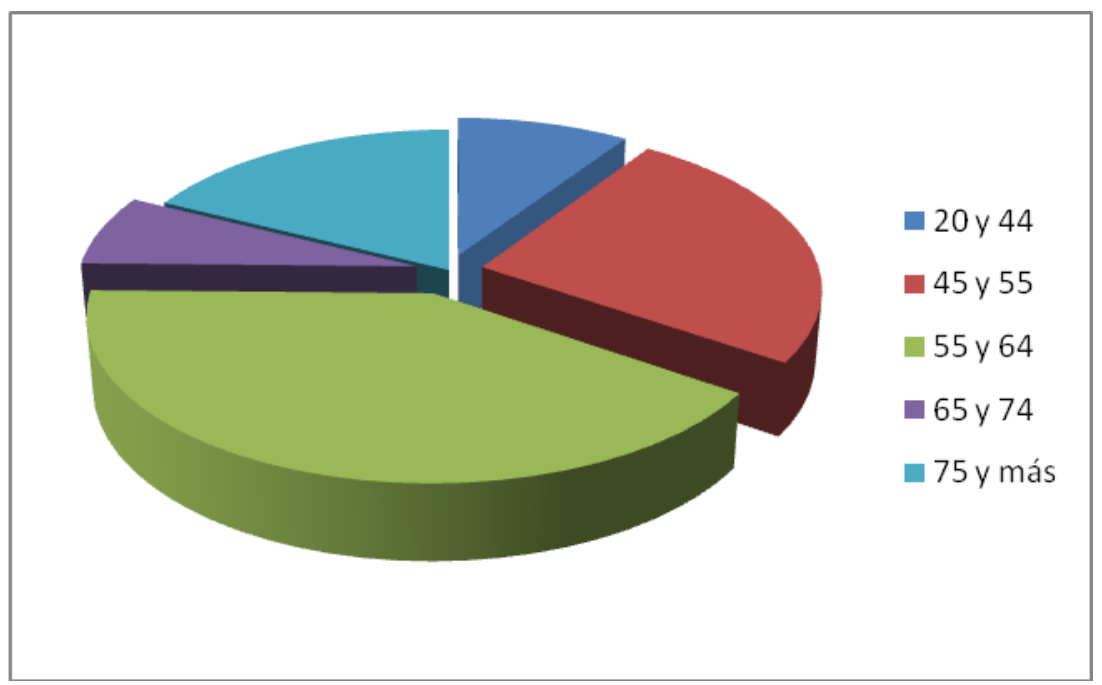

Figura 2: Distribución de los cuidadores según sexo y edad

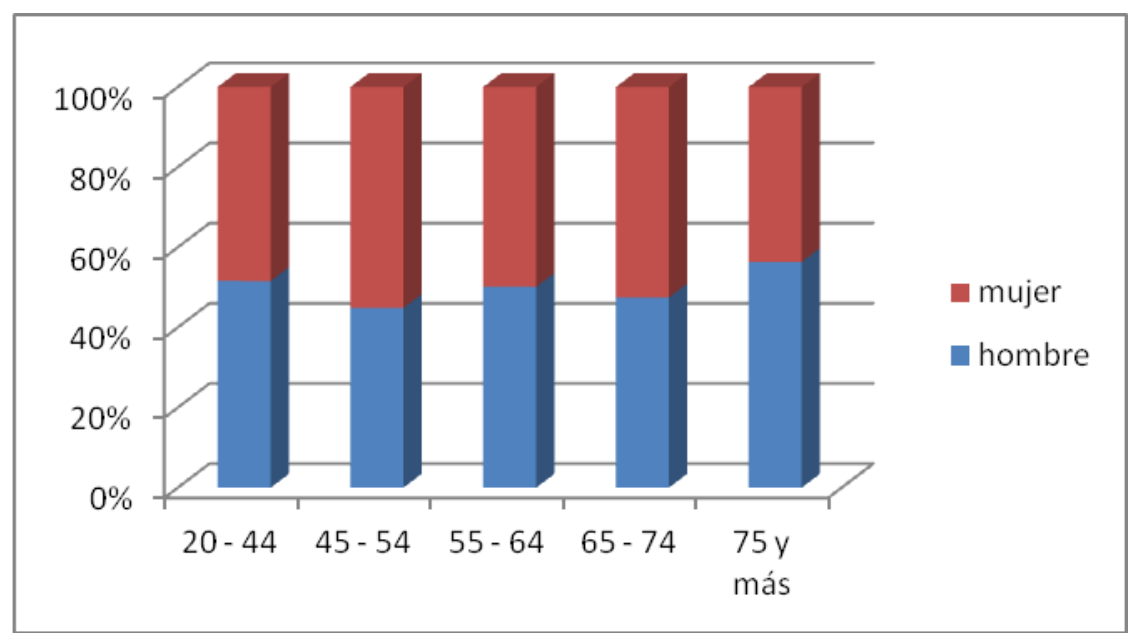

Tabla 1. Características socio-demográficos del cuidador, motivo de ingreso, nivel de dependencia del enfermo y estancia media.

\begin{tabular}{|c|c|c|c|}
\hline EDAD MEDIA & \multicolumn{3}{|c|}{ MOTIVO DE INGRESO } \\
\hline Hombre & $\begin{array}{l}60,73 \text { años } \\
\text { (DE }=13,53)\end{array}$ & $\begin{array}{l}\text { Enfermedad aguda } \\
\text { (reagudización) }\end{array}$ & $89,5 \%$ \\
\hline Mujer & $\begin{array}{l}59,02 \text { años } \\
(\mathrm{DE}=12,92)\end{array}$ & Enfermedad crónica & $10,5 \%$ \\
\hline $\begin{array}{l}\text { NIVEL DE } \\
\text { ESTUDIOS }\end{array}$ & \multicolumn{3}{|c|}{$\begin{array}{l}\text { NIVEL DE DEPENDENCIA } \\
\text { (BARTHEL) }\end{array}$} \\
\hline $\begin{array}{l}\text { No sabe leer, ni } \\
\text { escribir }\end{array}$ & $5,2 \%$ & Dependencia total & $27,7 \%$ \\
\hline $\begin{array}{l}\text { No ha cursado } \\
\text { estudios, pero sabe } \\
\text { leer y escribir }\end{array}$ & $16,1 \%$ & Dependencia grave & $8,2 \%$ \\
\hline Primarios & $52,1 \%$ & Dependencia moderada & $19,9 \%$ \\
\hline
\end{tabular}




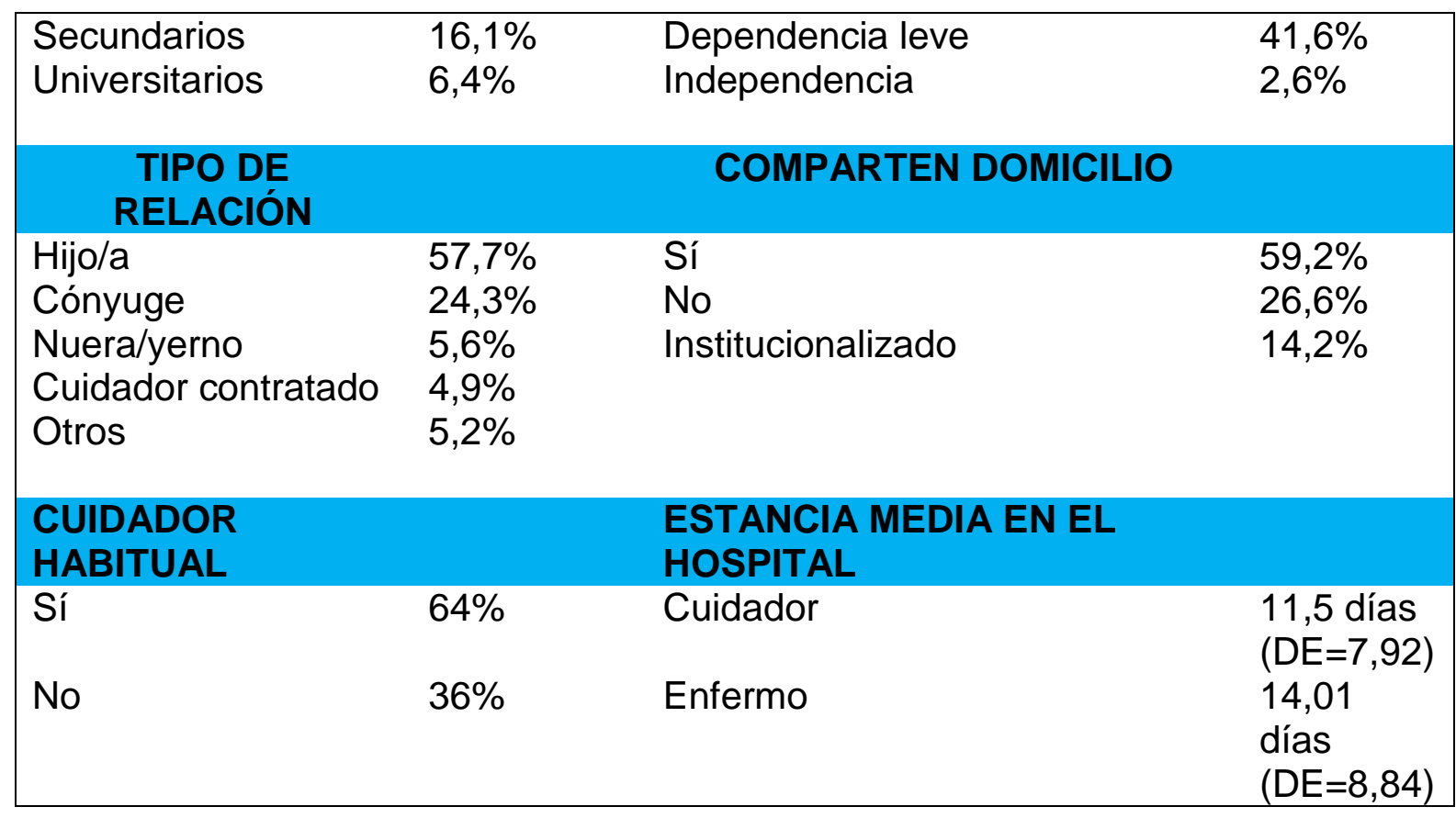

\section{Análisis de las dimensiones tangibilidad, fiabilidad, seguridad, capacidad de respuesta y empatía.}

En relación a la valoración de la dimensión "tangibilidad" y la dependencia $(p=0.021)$, se encuentran diferencias significativas. La correlación es muy baja y positiva $(p=0.141)$, de forma que a nivel de dependencia bajo, hay baja valoración de la "tangibilidad" y viceversa. Asimismo se obtienen diferencias significativas en la valoración de la dimensión "fiabilidad"; la puntúan más alto los cuidadores que comparten domicilio $(p=0.049)$ y también los cuidadores "sin estudios".

Las mujeres puntúan más alto que los hombres $(p=0.048)$ la dimensión "seguridad", igual que los cuidadores que no conviven con el enfermo ( $p=0.042)$. Los valores de "seguridad" son más altos también en el grupo de Estudios Primarios $(p<0.001)$. Por su parte, los valores de "capacidad de respuesta" son más altos en el grupo de Estudios Universitarios $(p<0.001)$. En la valoración de la dimensión "empatía" se hallan los siguientes diferencias significativas: los hombres la puntúan más alto que las mujeres $(p=0.001)$, los cuidadores que no comparten domicilio $(p=0.021)$, y los cuidadores con estudios secundarios y universitarios $(p=0.006)$.

No existe relación entre las valoraciones de las dimensiones y los días de estancia en el hospital, de los pacientes, ni el motivo de ingreso.

\section{Valoración de satisfacción con los cuidados y satisfacción general con la atención recibida según perfil socio-demográfico}

La puntuación obtenida en la cuestión de satisfacción global con los cuidados fue de $7,55(D E=1,254): 7,48(D E=1,40)$ en hombres y $7,57(D E=1,21)$ en mujeres. Con respecto a la satisfacción global con la atención recibida, la puntuación fue de 7,11 $(D E=1,245): 7,03(D E=1,080)$ en hombres y $7,14(D E=1,292)$ en mujeres.

En la Tabla 2 se expone la satisfacción global con la atención recibida y con los cuidados, según tipo de relación y sexo: 
- Tanto en mujeres como en hombres, la satisfacción global con los cuidados obtiene una puntuación un poco más alta $(0,44)$ que en la valoración de la satisfacción global con la atención recibida.

- Las puntuaciones en ambos sexos casi sin iguales.

- Todas las medias son muy similares (entre 7,03 - 7,48), y teniendo en cuenta que la escala de respuesta es de 1 a 10 , se pueden considerar puntuaciones altas.

- En la satisfacción con los cuidados, las medias oscilan entre 7,14 - 8,50.

- En la satisfacción con los cuidados, las medias oscilan entre 6,67 - 8,00.

- Hay una ligera diferencia en la puntuación de los cuidados (más alta).

- Siempre puntúa más alto la mujer, salvo en la valoración de los cuidados, cuando la valora el yerno, que adjudica una puntuación más alta.

- Donde más distancia hay entre la puntuaciones es en la valoración de los cuidados de los yernos $(8,50)$ y la valoración de la atención global del cuidador contratado $(6,75)$.

Tabla 2: Satisfacción global con la atención recibida y con los cuidados en cuidadores, según tipo de relación y sexo.

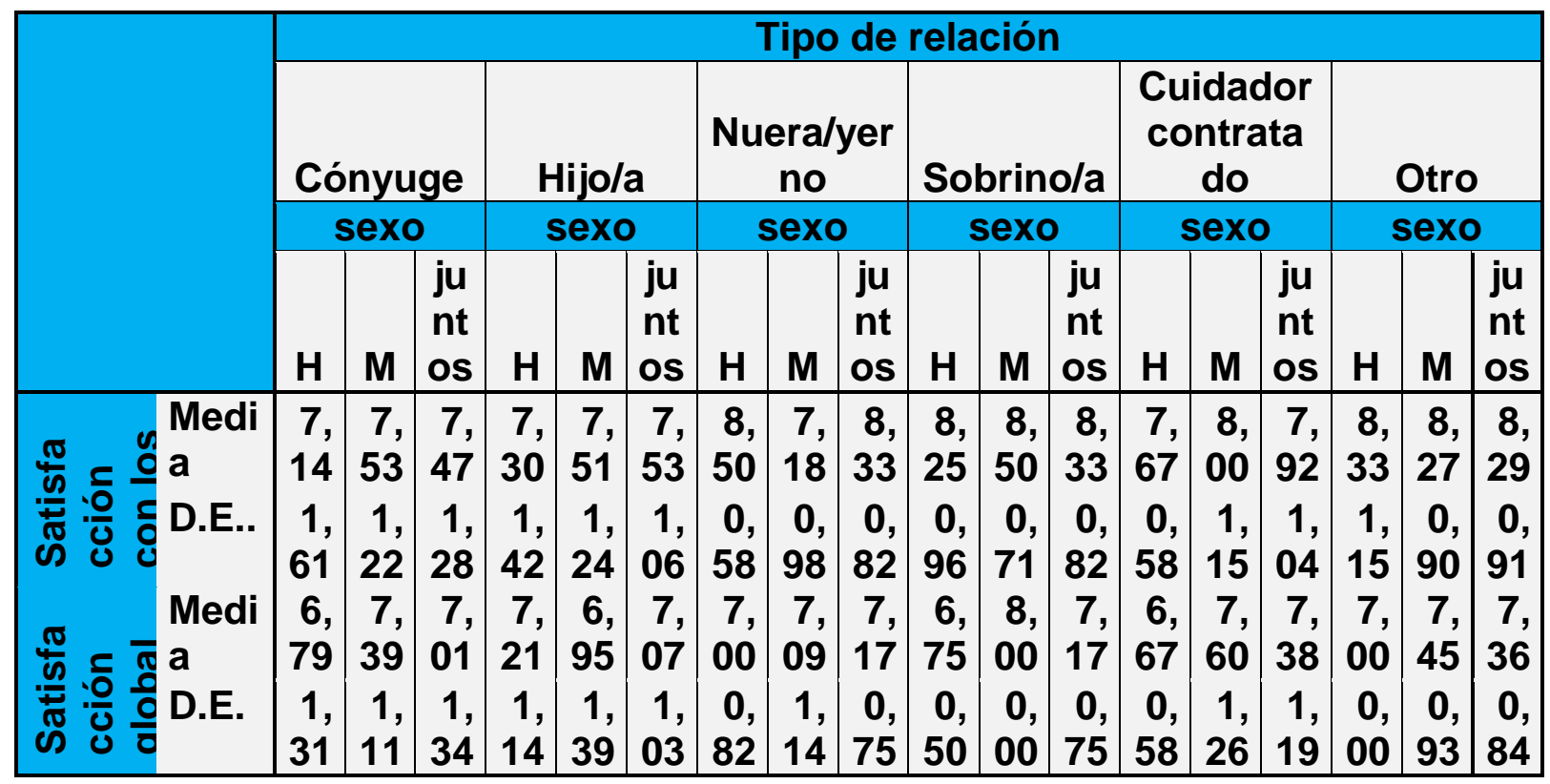

En la Tabla 3 se puede observar la puntuación en la satisfacción global con la atención recibida y con los cuidados según rango de edad.

- Los que otorgan una valoración algo más alta son los cuidadores más jóvenes y los más mayores.

- Los que más bajo valoraron los cuidados $(7,40)$ y la atención recibida $(6,84)$ fueron los cuidadores entre 45 y 54 años.

- Las puntuaciones más altas en la atención recibida $(7,59)$ las otorgaron las mujeres de más de 75 años.

- Las puntuaciones más altas en los cuidados $(8,75)$ las otorgaron los hombres entre 65 y 74 años.

- Los que menos satisfechos quedaron con la atención $(6,75)$ fueron los hombres entre 65 y 74 años, los mismos que dieron la puntuación más alta a los cuidados $(8,75)$. La diferencia es de 2 puntos. 
- Los menos satisfechos con los cuidados fueron los hombres entre 45 y 54 años.

Tabla 3. Satisfacción global con la atención recibida y con los cuidados, según sexo y rango de edad.

\begin{tabular}{|c|c|c|c|c|c|c|c|c|c|c|c|c|c|c|c|c|}
\hline & \multicolumn{15}{|c|}{ Edad del cuidador } \\
\hline & & \multicolumn{3}{|c|}{$\begin{array}{l}\text { 20- } 44 \\
\text { años }\end{array}$} & \multicolumn{3}{|c|}{$\begin{array}{l}45-54 \\
\text { años }\end{array}$} & \multicolumn{3}{|c|}{$\begin{array}{c}55 \text { y-64 } \\
\text { años }\end{array}$} & \multicolumn{3}{|c|}{ 65-74 años } & \multicolumn{3}{|c|}{$\begin{array}{c}\text { Más de } 75 \\
\text { años }\end{array}$} \\
\hline & & $\mathbf{H}$ & $\mathbf{M}$ & $\begin{array}{l}\text { Jun } \\
\text { tos }\end{array}$ & $\mathbf{H}$ & $\mathbf{M}$ & $\begin{array}{l}\text { Jun } \\
\text { tos }\end{array}$ & $\mathbf{H}$ & $\mathbf{M}$ & $\begin{array}{l}\text { Jun } \\
\text { tos }\end{array}$ & H & $\mathbf{M}$ & $\begin{array}{l}\text { Jun } \\
\text { tos }\end{array}$ & $\mathbf{H}$ & $\mathbf{M}$ & $\begin{array}{l}\text { Jun } \\
\text { tos }\end{array}$ \\
\hline $\begin{array}{l}\text { Satisfa } \\
\text { cción } \\
\text { con los }\end{array}$ & $\begin{array}{l}\text { Me } \\
\text { dia } \\
\text { D.E }\end{array}$ & $\begin{array}{r}7, \\
17\end{array}$ & $\begin{array}{r}8, \\
11\end{array}$ & $\begin{array}{r}7,8 \\
8\end{array}$ & $\begin{array}{r}6, \\
92\end{array}$ & $\begin{array}{r}7, \\
52\end{array}$ & $\begin{array}{r}7,4 \\
0\end{array}$ & $\begin{array}{r}7, \\
6\end{array}$ & $\begin{array}{r}7 \\
44\end{array}$ & $\begin{array}{r}7,4 \\
8\end{array}$ & $\begin{array}{r}8 \\
75 \\
\end{array}$ & $\begin{array}{r}7, \\
27\end{array}$ & $\begin{array}{r}7,5 \\
8\end{array}$ & $\begin{array}{r}7 \\
54\end{array}$ & $\begin{array}{r}7,8 \\
2\end{array}$ & 7,74 \\
\hline $\begin{array}{l}\text { cuida } \\
\text { dos }\end{array}$ & & $\begin{array}{r}0, \\
98\end{array}$ & $\begin{array}{r}1,3 \\
3\end{array}$ & $\begin{array}{r}1,3 \\
0\end{array}$ & $\begin{array}{r}1, \\
66\end{array}$ & $\begin{array}{r}1, \\
08\end{array}$ & $\begin{array}{r}1,2 \\
2\end{array}$ & $\begin{array}{r}1, \\
35\end{array}$ & $\begin{array}{r}1, \\
28\end{array}$ & $\begin{array}{r}1,3 \\
0\end{array}$ & $\begin{array}{r}0, \\
5\end{array}$ & $\begin{array}{r}1, \\
33\end{array}$ & $\begin{array}{r}1,3 \\
5\end{array}$ & $\begin{array}{r}1, \\
39\end{array}$ & $\begin{array}{r}1,0 \\
3\end{array}$ & 1.13 \\
\hline Satisfa & Me & 7, & 7, & 7,3 & 6 , & 6 , & 6,8 & 7 , & 7 , & 7,1 & 6 & 6 , & 6,8 & & 7,5 & \\
\hline $\begin{array}{l}\text { cción } \\
\text { global } \\
\text { con la } \\
\text { aten }\end{array}$ & $\begin{array}{l}\text { dia } \\
\text { D.E }\end{array}$ & 17 & 37 & 2 & 85 & 83 & 4 & 16 & 13 & 4 & 75 & 93 & 9 & 7 & 9 & 7,43 \\
\hline $\begin{array}{l}\text { recibi } \\
\text { da }\end{array}$ & & $\begin{array}{l}0, \\
75\end{array}$ & $\begin{array}{l}1, \\
5\end{array}$ & $\begin{array}{r}1,3 \\
5\end{array}$ & $\begin{array}{r}0, \\
9\end{array}$ & $\begin{array}{r}1, \\
36\end{array}$ & $\begin{array}{r}1,2 \\
7\end{array}$ & $\begin{array}{r}1, \\
14\end{array}$ & $\begin{array}{l}1, \\
3\end{array}$ & $\begin{array}{r}1,2 \\
6\end{array}$ & $\begin{array}{r}0 \\
96\end{array}$ & $\begin{array}{r}0, \\
96\end{array}$ & $\begin{array}{r}0,9 \\
4\end{array}$ & $\begin{array}{r}1, \\
35\end{array}$ & $\begin{array}{r}1,0 \\
8\end{array}$ & 1,17 \\
\hline
\end{tabular}

En cuanto a la satisfacción según niveles de dependencia de los pacientes (Tabla 4), los resultados son similares: existe una ligerísima diferencia entre la valoración general y la valoración con los cuidados. Los que mejor valoran los cuidados son los cuidadores de los pacientes independientes, y los que peor aquellos con pacientes con dependencia grave a su cargo. Los que mejor valoran los cuidados y peor la atención en general son los cuidadores de los pacientes independientes.

Tabla 4. Satisfacción según el nivel de dependencia (Barthel) del paciente.

\begin{tabular}{|c|c|c|c|c|c|c|}
\hline & \multicolumn{5}{|c|}{ Nivel de dependencia } \\
\hline & & $\begin{array}{c}\text { Depen } \\
\text { dencia } \\
\text { total }\end{array}$ & $\begin{array}{l}\text { Depen } \\
\text { dencia } \\
\text { severa }\end{array}$ & $\begin{array}{l}\text { Dependen } \\
\text { cia } \\
\text { moderada }\end{array}$ & $\begin{array}{c}\text { Depen } \\
\text { dencia } \\
\text { leve }\end{array}$ & $\begin{array}{c}\text { Indepen } \\
\text { dencia }\end{array}$ \\
\hline $\begin{array}{l}\text { Satisfacción } \\
\text { con los } \\
\text { cuidados } \\
\text { Satisfacción } \\
\text { global con } \\
\text { la atención } \\
\text { recibida }\end{array}$ & $\begin{array}{l}\text { Me } \\
\text { dia } \\
\text { D.E. } \\
\text { Me } \\
\text { dia } \\
\text { D.E. }\end{array}$ & $\begin{array}{r}7,8 \\
1,2 \\
7,2 \\
1,2\end{array}$ & $\begin{array}{r}7,2 \\
1,7 \\
\\
6,8 \\
1,7\end{array}$ & $\begin{array}{r}7,4 \\
1,4 \\
\\
7,1 \\
1,2\end{array}$ & $\begin{array}{r}7,5 \\
1,1 \\
7,2 \\
1,2\end{array}$ & \begin{tabular}{c|}
7,98 \\
0,9 \\
\\
6,6 \\
1,7
\end{tabular} \\
\hline
\end{tabular}

Finalmente, si se analiza la satisfacción teniendo en cuenta los niveles de estudios (Tabla 5), los resultados muestran una ligera diferencia entre las puntuaciones de los cuidadores con el nivel de estudios universitarios, con los cuidadores de nivel de estudios secundarios, en cuanto a la valoración del cuidado. Se sigue con la tendencia de valorar un poco peor la atención general. 
Tabla 5. Satisfacción general y con la atención, según nivel de estudios.

\begin{tabular}{|c|c|c|c|c|c|c|}
\hline & \multicolumn{5}{|c|}{ Nivel de estudios } \\
\hline & & $\begin{array}{l}\text { No sabe leer, } \\
\text { ni escribir }\end{array}$ & $\begin{array}{c}\text { No ha } \\
\text { cursado } \\
\text { estudios, } \\
\text { pero sabe } \\
\text { leer y } \\
\text { escribir }\end{array}$ & $\begin{array}{c}\text { Prima } \\
\text { rios }\end{array}$ & $\begin{array}{l}\text { Secunda } \\
\text { rios }\end{array}$ & $\begin{array}{l}\text { Universita } \\
\text { rios }\end{array}$ \\
\hline $\begin{array}{l}\text { Satis } \\
\text { facción } \\
\text { con los } \\
\text { cuidados }\end{array}$ & $\begin{array}{l}\text { Me } \\
\text { dia }\end{array}$ & 7,4 & 7,7 & 7,6 & 7,3 & 7,8 \\
\hline $\begin{array}{l}\text { Satis } \\
\text { facción } \\
\text { global } \\
\text { con la } \\
\text { atención } \\
\text { recibida }\end{array}$ & $\begin{array}{l}\text { D.E. } \\
\text { Me } \\
\text { dia } \\
\text { D.E. }\end{array}$ & $\begin{array}{l}1,5 \\
7,1 \\
1,3\end{array}$ & $\begin{array}{l}1,3 \\
7,4\end{array}$ & $\begin{array}{l}1,2 \\
7,1 \\
1,3\end{array}$ & 6,9 & 7,1 \\
\hline
\end{tabular}

\section{DISCUSIÓN}

Este trabajo analiza las influencias del perfil socio-demográfico con la satisfacción de los cuidadores. En este sentido, hay muy pocos estudios que exploren el impacto de las variables socio-demográficas de los cuidadores de los enfermos geriátricos hospitalizados y su nivel de satisfacción.

El perfil del cuidador, en cuanto al género y la edad, es muy similar a otros estudios relacionados con los cuidadores; mujer, alrededor de 55 años, que no trabaja fuera del domicilio y con estudios primarios ${ }^{10-12}$. En cuanto al nivel de educación, se puede destacar que en el presente trabajo se encontró una proporción bastante alta $(21,3 \%)$ de personas que no han cursado ni estudios básicos. Esto es destacable porque en España la tasa de analfabetismo es de $2,6 \%$ y la población sin estudios de $12,8 \%{ }^{13-}$ 14.

En cuanto al análisis del las cinco dimensiones del instrumento aplicado, se puede encontrar que la "tangibilidad" es muy importante para los cuidadores con enfermos de dependencia significativa, ya que mientras más dependientes sean los enfermos peor se valoran las cosas tangibles. Estos cuidadores se encuentran muchas veces con dificultades para el aseo y para la movilización de los pacientes si los espacios son reducidos y las instalaciones no están adaptados a las necesidades de los enfermos. Estudios sobre la tangibilidad de los centros donde se atienden personas de avanzada edad demuestran que están más satisfechos con la atención en edificaciones nuevas y luminosas ${ }^{15}$. En el ambiente hospitalario se debe encontrar una estructura física del edificio, como del mobiliario, en buenas condiciones de apariencia agradable ${ }^{16-17}$.

Las diferencias estadísticamente significativas en la dimensión "fiabilidad" se obtuvieron relacionadas con los cuidadores que comparten domicilio. Esto no debe causar sorpresa, ya que estos cuidadores son los que realmente conocen las 
dificultades añadidas del trabajo con personas de avanzada edad, pues conviven con ellos las 24 horas del día. También se encontró el hallazgo de que esta dimensión la valoran más los cuidadores "sin estudios". Otros estudios parecidos encontraron que el nivel educativo y el sexo fueron determinantes de la satisfacción de los pacientes con la atención de enfermería ${ }^{18-19}$.

La "capacidad de respuesta" es más valorada en el grupo de Estudios Universitarios. Algunos estudios relacionan el nivel de estudios con la disconformidad ${ }^{20}$. El reto de la enfermería es ofrecer cuidado y su continuidad. Seguramente que esta situación habrá que asumirla con más visibilidad ${ }^{21}$. En cuanto a la "seguridad", las diferencias encontradas tienen que ver con el género (la mujer puntúa más alto). Los cuidadores que no conviven con el enfermo también lo puntúan más alto, y los cuidadores "sin estudios" en el grupo con estudios primarios. Algunos estudios parecidos encontraron que el nivel educativo y el género fueron determinantes de la satisfacción de los pacientes con la atención de enfermería ${ }^{18-19}$, pero no hacen referencia si encuentran diferencias en relación a la seguridad.

En la dimensión "empatía", los hombres están más satisfechos con esta dimensión que las mujeres; también puntúan más en esta dimensión los cuidadores que no comparten domicilio. Los que peor valoran esta dimensión son los cuidadores con estudios universitarios. El tipo de trato y la información durante la hospitalización aparece en la bibliografía consultada ${ }^{9}$ como una de las necesidades a las que se debe prestar una mayor atención por parte de los profesionales. Algunos autores resaltan la importancia que tiene tanto para el paciente como para el cuidador recibir una adecuada información por parte del personal de enfermería sobre su tratamiento. Los contactos frecuentes y la comunicación con los consumidores se perciben como una de las garantías de calidad de la atención ${ }^{19}$. El trato humano recibido por parte del personal sanitario, destacando la empatía, la información transmitida de forma comprensible y el tiempo dedicado por el profesional a la relación personal con la persona a la que atiende, así como la posibilidad de ésta para expresarse, son los principales factores que influyen en la satisfacción con la atención recibida, así como el hecho de que se tengan en cuenta los intereses y las expectativas de la persona ${ }^{22}$.

Los datos obtenidos en este estudio ponen de manifiesto que no existe relación entre las valoraciones de las dimensiones y los días de estancia en el hospital de los pacientes, ni el motivo de ingreso. Aunque existen estudios que evalúan las 5 dimensiones de SERVQUAL ${ }^{23-26}$, ninguno de ellos analiza significación estadística con estas variables.

Si se analiza la satisfacción global con la atención y con los cuidados, los resultados se encuentran entre "mediatamente buenos", siempre obteniendo una puntuación ligeramente más alta en la valoración de los cuidados. Este hallazgo es muy similar a estudios parecidos realizados en diferentes ámbitos ${ }^{19}$. En cuanto a la satisfacción, la mujer siempre puntúa ligeramente más alto, mientras en otros estudios parecidos se encuentra que la mujer puntuó más bajo ${ }^{19}$. Las diferencias halladas en la valoración de la satisfacción global, tanto con los cuidados como con la atención global, según las variables socio-demográficas, son muy pequeñas. La máxima diferencia se encuentra entre la valoración de los cuidados de los yernos $(8,50)$ y la valoración de la atención global del cuidador contratado $(6,75)$. La diferencia es 1,75 , y en una escala de 0 a 10 significa un 17\%, que se considera de mínima importancia. No se pudo contrastar este resultado, ya que no se encontraron otros estudios parecidos. 
Aunque con una muestra de 267 cuidadores es suficiente para obtener conclusiones generales, hay que tener presente que no se puede extrapolar el resultado a nivel nacional, ni internacional.

\section{CONCLUSIONES}

En el mundo actual, las expectativas de los pacientes y de los cuidadores se deben tener en cuenta para poder ofrecer un cuidado de calidad. En la práctica se cuenta con la opinión del cuidador, pero hay falta de evidencia científica en este aspecto. Conocer la percepción que tienen los cuidadores sobre el cuidado y su satisfacción en general es un indicador de calidad muy importante. Las razones de la insatisfacción deben ser estudiadas y resueltas. Pacientes y cuidadores satisfechos son esenciales para la supervivencia de las instituciones sanitarias. Los usuarios que están satisfechos con los servicios, no sólo elegirán el mismo hospital la próxima vez, sino que también pueden influir en las preferencias de otros pacientes.

Existe una necesidad de aumentar el nivel de conocimiento sobre los cuidadores y conocer sus expectativas sobre el cuidado para elaborar estrategias de cuidado acordes con sus preferencias. Sería muy importante tener en cuenta los factores que se identifiquen como influyentes en la calidad de atención a los enfermos geriátricos. Por este motivo, los factores analizados en el presente estudio (género, edad, nivel de estudios del cuidador, tiempo de hospitalización, nivel de dependencia del enfermo, convivencia continua, e ingresos anteriores) son muy relevantes. La satisfacción general con la atención y con los cuidados prestados, según los cuidadores, se caracteriza por tener un nivel "medianamente bueno" en nuestro estudio.

El conocimiento de las deficiencias detectadas sobre el cuidado puede proporcionar a los profesionales pistas sobre dónde se debe intervenir para conseguir cambios favorables para aumentar la satisfacción. El presente estudio se pretendió dotar con suficiente validez para que sirva de aplicación para futuras intervenciones e investigaciones. Este trabajo está en la línea con las actuales investigaciones sobre la mejora de cuidados y en la búsqueda de la excelencia de cuidados. Los resultados pueden ser de gran interés científico por la escasez de estudios similares en el ámbito de las Ciencias de la Salud.

\section{REFERENCIAS}

1. WHO. Cifras y datos sobre el envejecimiento de la población, Abril 2012. [Citado 2016 Abr 04]. Disponible en: http://www.who.int/features/factfiles/ageing/ageing facts/es/datos

2. Organizacion Mundial de la Salud. Informe mundial sobre la discapacidad. Malta, 2011. [Citado 2016 Abr 02]. Disponible en: http://www.who.int/iris/bitstream/10665/75356/1/9789240688230 spa.pdf.

3. Ministerio de Sanidad, Servicios Sociales e Igualdad del Sistema Nacional de Salud. Madrid, 2012. [citado 2016 Abr 04]. Disponible en: http://www.msc.es/organizacion/sns/docs/sns2012/SNS012.pdf.

4. Pujol Rodríguez R, Abellán García A. Un perfi I de las personas mayores en España, 2015. Indicadores estadísticos básicos". Madrid, Informes Envejecimiento en red $n^{\circ}$ 10. [Fecha de publicación: 22/01/2015]. [Citado 2016 Abr 04]. 
Disponible en: http://envejecimiento.csic.es/documentos/documentos/enredindicadoresbasicos 15.pdf>

5. Unidad de Pacientes Pluripatológicos: Estándares y Recomendaciones Informes. Estudios e investigación. Madrid: Edit. Ministerio de Sanidad y Política Social, 2009.

6. OMS. Serie de Informes Técnicos: Dieta, nutrición y prevención de enfermedades crónicas. Informe de una Consulta Mixta de Expertos OMS/FAO. Ginebra: Organización Mundial de la Salud, 2003. [Citado 2016 Abr 04]. Disponible en :http://www.fao.org/3/a-ac911s.pdf

7. Millán Calenti C. Gerontología y Geriatría; valoración e intervención. Intervención socio-laboral y familiar. Madrid: Editorial Médica Panamericana, 2011.

8. Ministerio de Trabajo y Asuntos Sociales. Atención a las personas en situación de dependencia en España. Libro Blanco. Madrid: Ministerio de Trabajo y Asuntos Sociales e Instituto de Mayores y Servicios Sociales. [Citado 2016 Abr 02] Disponible https://www.uab.cat/Document/580/416/LibroBlancoDependencia 01.pdfMinisterio

9. Hanzeliková Pogrányivá A. La calidad percibida de los cuidados por los pacientes geriátricos agudos y los cuidadores principales. Reduca Trabajos Fin de Master 2011; 3 (2): 67-82.

10. Mendoza Sánchez RM, Hernández García EL, Medina Pérez M, Company Sancho MC, Gómez Perera M L, Estrada Suárez Pérez LS, et al. Perfil del cuidador principal en el área de salud de Gran Canaria. Ene. [revista en la Internet]. 2014 [citado 2016 Abr 04]; 8(2): Disponible en: http://scielo.isciii.es/scielo.php?script=sci_arttext\&pid=S1988-

348X2014000200002\&lng=es. http://dx.doi.org/10.4321/S1988348X2014000200002.

11. Muíño Joga M, Vidal Fernández M, Rodríguez Rodríguez E, Hermida Rodríguez A, Herves Riego E. Perfil sociocultural de los cuidadores de pacientes inmovilizados en Atención Primaria. Cuadernos de Atención Primaria 2009; 16 : 280-283.

12. Bermejo Higuera JC, Villacieros Durbán M, Carabias Maza R, Lozano González B. Niveles y motivos de satisfacción o insatisfacción en los familiares de los fallecidos en una unidad de cuidados paliativos. Gerokomos 2014; 25: 111-114.

13. Abellán García A, Viches Fuentes J, Pujol Rodriguez R. Informe de envejecimiento en red 2014. Un perfil de las personas mayores en España. Indicadores estadísticos básicos, 2014. [Citado 2016 Abr 04]. Disponible en: http://sienacoop.org/2014/02/18/un-per\%EF\%AC\%811-de-las-personas-mayoresen-espana-2014-indicadores-estadisticos-basicos/

14. Instituto Nacional de Estadística. España al comienzo del siglo XXI: Educación y Estudios. [Citado 2016 Abr 04]. Disponible en: file:///C:/Users/Usuario/Downloads/pags28-35\%20(1).pdf

15. Forgan Morle KM. Patient satisfaction: care of the elderly J Adv Nurs.1984; 9: 7176.

16. Cedrés de Bello S. Humanización y Calidad de los Ambientes Hospitalarios. RFM 2000; 23: 93-97. [Citado 2016 Abr 04]. Disponible en: http://www.scielo.org.ve/scielo.php?script=sci arttext\&pid=S0798$04692000000200004 \&$ ing $=$ es.

17. Calidad de atención en salud; percepción de los usuarios Hospital Universitario del Valle. [Citado 2016 Abr 04]. Disponible en: http://www.fundacionfundesalud.org/pdffiles/calidad-de-la-atencion-en-salud.pdf 
18. Torres Contreras C. Percepción de la calidad del cuidado de enfermería en pacientes hospitalizados. Av Enferm 2010; 28: 98-110. [Citado 2016 Abr 02] Disponible en http://www.scielo.org.co/scielo.php?script=sci arttext\&pid=S0121$45002010000200009 \& \operatorname{lng}=\mathrm{en}$.

19. Hala YS, Hoda AM, Esraa EM. Patients' Perceptions As Indicators of Quality of Nursing Service Provided At Al Noor Specialist Hospital at Makkah Al Moukarramah, KSA. J Am Sci 2013; 9: 71-78.

20. Johansson $P$, Olèni $M$, Fridlund $B$. Patient satisfaction with nursing in context of health care: a literature study. Scand J Caring Sci 2002; 16: 337-244.

21. Corrales Nevado D, Palomo Cobos L. la importancia de la longitudinalidad, integralidad, coordinación y continuidad de los cuidados efectuados por enfermería. Enf Clin 2014; 24:1.

22.Bonill de las Nieves C. La importancia de las habilidades comunicativas en la humanización de los cuidados. Index Enferm 2008; 17: 74-75. [Citado 2016 Abr 04] . Disponible en: http://scielo.isciii.es/scielo.php?script=sci arttext\&pid=S1132$12962008000100017 \&$ lng $=$ es.

23. Senarath U, Gunawardena NS, Sebastiampillai B, Senanayake A, Lekamge S, Seneviratna A, Wijeratne D. Patient satisfaction with nursing care and related hospital services at the National Hospital of Sri Lanka. Leadership in Health Services 2013; 26: 63-77.

24. Ramez WS. Patients' Perception of Health Care Quality, Satisfaction and Behavioral Intention: An Empirical Study in Bahrain. International Journal of Business and Social Science 2012; 3 (18): 131-141.

25. Rahman MS, Khan AH, Haque MM. A conceptual study on the relationship between service quality towards customer satisfaction: Servqual and Gronroos's service quality model perspective. Asian Social Science 2012; 8 (13): 201.

26. Purcărea VL, Gheorghe IR, Petrescu CM. The assessment of perceived service quality of public health care services in Romania using the SERVQUAL scale. Procedia Economics and Finance 2013; 6: 573-585.

Recibido: 8 de febrero 206;

Aceptado: 15 de abril 2016

ISSN 1695-6141

( ) COPYRIGHT Servicio de Publicaciones - Universidad de Murcia 